\title{
On the Julia Directions of the Value Distribution of Nondegenerate Transendental Holomorphic Maps of $\mathbf{C}^{2}$ to $\mathbf{C}^{2}$
}

\author{
Yukinobu Adachi ${ }^{1}$ \\ ${ }^{1}$ Nishinomiya City, Kurakuen 2-bannchyo, Japan \\ Correspondence: Yukinobu Adachi, Nishinomiya City, Kurakuen 2-bannchyo, Japan. Tel: 81-798-717-391.
}

Received: February 19, 2013 Accepted: May 6, 2013 Online Published: June 28, 2013

doi:10.5539/jmr.v5n3p8

URL: http://dx.doi.org/10.5539/jmr.v5n3p8

\begin{abstract}
We prove that for a nondegenerate holomorphic map $F=(f(x, y), g(x, y))$ of $\mathbf{C}^{2}$ to $\mathbf{C}^{2}$ where $f$ and $g$ are entire functions and $f$ is a transendental one, there exists a ray $J(\theta)=\left\{(x, y) ; x=t e^{i \theta}, y=k t e^{i \theta}(0 \leqq t<\infty)\right\}$ where $k$ is an arbitrarily fixed complex number except some Lebesgue measure zero set and $\theta$ is some real number depending on value $k$, such that $F(x, k x)$, in any open cone in $\mathbf{C}^{2}$ with vertex $(0,0)$ containing the ray $J(\theta)$, does not omit any algebraic curve with three irreducible components in a general position.
\end{abstract}

Keywords: Julia directions, equi-two-dimensional nondegenerate holomorphic maps, Little Picard theorem, Montel's theorem

2000 Mathmatics Subject Classification: Primary 32H02; Secondary 32H30, 32H25 and 32 Q45

\section{Introduction}

Using the theory of normal family, G. Julia in 1919 improved the big and little Picard theorem such that for every transendental holomorphic function has a Julia direction as the following theorem.

Theorem Let $f(z)$ be a transendental entire function on $\mathbf{C}$. Then there exists a ray $J(\theta)=\left\{z=t e^{i \theta} ; 0 \leqq t<\infty\right\}$ such that $f$, in any open sector with vertex $z=0$ containing the ray $J(\theta)$, takes every value in $\mathbf{C}$ except at most one value in infinite times.

Z-H. Tu in 1996 proved a generaization for a transendental entire holomorphic curve with an asymptotic value in $\mathbf{P}^{n}$.

We shall prove a generalization for a nondegenerate (that is, the image set contains an open set) transendental entire map of $\mathbf{C}^{2}$ to $\mathbf{C}^{2}$ (Theorem 4.1) by the method of Kobayashi hyperbolic geometry (for the fundamental concepts and properties, see Kobayashi,1998).

\section{Preliminary (1)}

Theorem 2.1 (Julia, 1919, p. 103) Let $f(z)$ be a holomorphic function on $R \leqq|z|<\infty$ and $z=\infty$ is an essential singular point of $f$. Then there is a half-line $L_{\theta_{0}}:=\left\{z=\right.$ Rte $\left.e^{i \theta_{0}}\right\}$ where $\theta_{0}$ is some real number and $t \in[1, \infty)$ such that for every $\varepsilon>0 f(z)$ takes all values except at most one value in infinite times in $G$ where $G:=\bigcup_{\left|\theta-\theta_{0}\right|<\varepsilon} L_{\theta}$.

To prove above the theorem, the following lemma is essential.

Lemma 2.2 We set $f_{t}=f(z t)$ for $t \in[1, \infty)$. We consider $\left\{f_{t}\right\}_{t \in[1, \infty)}$ a family of holomorphic functions on $R \leqq|z|<$ $\infty$. Then $\left\{f_{t}\right\}_{t \in[1, \infty)}$ is not normal in $\left\{R_{1}<|z|<R_{2}\right\}$ where $R_{1}$ and $R_{2}$ are some positive number with $R<R_{1}<R_{2}$. More pricisely there exists a sequence $\left\{f_{t_{i}}\right\}_{i=1,2, \cdots}$ for $t_{1}<t_{2}<\cdots \rightarrow \infty$ of the family has never convergent subsequence to a holomorphic function or $\infty$ in $\left\{R_{1}<|z|<R_{2}\right\}$.

By the method of blowing up at $(0,0)$, the following proposition is easy to see by Proposition 11.8 in (Adachi,1995).

Proposition 2.3 Let $f(x, y)$ be a transendental entire function. We denote by $E$ the set of $k \in \mathbf{C}$ such that $f(x, k x)$ is not transendental, then Lebesgue measure of $E$ (in $\mathbf{R}^{2}$ ) is zero.

\section{Preliminary (2)}

Following sections let $A$ be an algebraic curve with three irreducible components in $\mathbf{C}^{2}$ and $L_{\infty}$ is the line at infinity. 
From Theorem 8.1 in (Adachi, 1995), we have the following proposition.

Proposition 3.1 There are only following two cases:

(1) $S_{\mathbf{C}^{2}-A}\left(\mathbf{P}^{2}\right)=\emptyset$ or an algebraic curve $C$ in $\mathbf{P}^{2}$ which consists of irreducible components called a nonhyperbolic curve with respect to $A \cup L_{\infty}$ (for the prisice definition of the nonhyperbolic curve, see Definition 4.1 in Adachi, 1995),

(2) $S_{\mathbf{C}^{2}-A}\left(\mathbf{P}^{2}\right)=\mathbf{P}^{2}$, where $S_{\mathbf{C}^{2}-A}\left(\mathbf{P}^{2}\right)$ is roughly speaking the limitting degeneration locus of $d_{\mathbf{C}^{2}-A}$ (for the prisice definition, see Definition 1.1 in Adachi, 1995).

From Proposition 8.2 and Corollary 8.3 in (Adachi, 1995), it is concretely determined that when the case (2) above occurs and such cases are rather special ones.

Definition 3.2 When $A$ is an algebraic curve with three irreducible components in $\mathbf{C}^{2}$ and satisfies the case (1) in the above proposition, we call $A$ in a general position in $\mathbf{C}^{2}$.

From Theorem 12.1 in (Adachi, 1995), we have the following proposition.

Proposition 3.3 Let $A$ be an algebraic curve with three irreducible components in a general position in $\mathbf{C}^{2}$ and $F: \mathbf{C}^{2} \rightarrow \mathbf{C}^{2}-A$ be a holomorphic map with essential singularity at $L_{\infty}$. Then $F\left(\mathbf{C}^{2}\right) \subset C_{0}$ which is one of the irreducible components of $C$ in Proposition 3.1 and $C_{0} \nsubseteq A$. Therefore every nondegegenerate transendental entire map $F$ does not omit A.

From Theorem 4.4 in (Adachi, 1997), we have the following proposition.

Proposition 3.4 Let $A$ and $C$ be the same of above proposition. Then $\mathbf{C}^{2}-A$ is tautly imbedded modulo $S_{\mathbf{C}^{2}-A}\left(\mathbf{P}^{2}\right)(\subset$ $\left.C \cup L_{\infty}\right)$ in $\mathbf{P}^{2}$, that is, each sequence $\left\{F_{j}\right\}$ in $\operatorname{Hol}\left(\Delta^{k}, \mathbf{C}^{2}-A\right)$ where $\Delta^{k}=\Delta \times \cdots \times \Delta$ and $\Delta$ is the unit disk, we have one of the following:

(a) $\left\{F_{j}\right\}_{j=1,2, \ldots}$ has a subsequence which converges in $\mathrm{Hol}\left(\Delta^{k}, \mathbf{P}^{2}\right)$ with compact open topology;

(b) for each compact set $K \subset \Delta^{k}$ and each compact set $L \subset \mathbf{P}^{2}-S_{\mathbf{C}^{2}-A}\left(\mathbf{P}^{2}\right)$, there exists an integer $N$ such that $F_{j}(K) \cap L=\emptyset$ for $j \geqq N$, that is, $\left\{F_{j}\right\}$ diverges compactly to $S_{\mathbf{C}^{2}-A}\left(\mathbf{P}^{2}\right)$.

\section{Conclusion}

Theorem 4.1 Let $F=(f(x, y), g(x, y))$ be a nondegenerate holomorphic map of $\mathbf{C}^{2}$ to $\mathbf{C}^{2}$ where $f$ and $g$ are entire functions and $f$ is a transendental one, there exists a ray $J(\theta)=\left\{(x, y)=\left(t e^{i \theta}, k t e^{i \theta}\right)(0 \leqq t<\infty)\right\}$, where $k$ is an arbitrarily fixed complex number except some Lebesgue measure zero set and $\theta$ is some real number depending on the value $k$ such that $F(x, y)$, in any open cone in $\mathbf{C}^{2}$ with vertex $(0,0)$ containing the ray $J(\theta)$, does not omit any algebraic curve in $\mathbf{C}^{2}$ with three irreducible components in a general position.

Proof. Assume that $F$ omits an algebraic curve in $\mathbf{C}^{2}$ with three irreducible components in a general position. Let $D$ be a domain $D:=\{R \leqq|x|<\infty\}$ and $F$ be a restriction anew of $F$ to $\{y=k x, x \in D\}$. Let $E$ be the same of Proposition 2.3 and $k$ be fixed such as $k \notin E$. We set $F_{t}=(f(x t, k x t), g(x t, k x t))$ where $t \in[1, \infty)$. It is easy to see that $F_{t}(D) \subset F(D)$. From Lemma 2.2 and Proposition $2.3\left\{f\left(x t_{i}, k x t_{i}\right)\right\}_{i=1,2, \cdots}$ has no convergent subsequence in $\left\{R_{1}<|x|<R_{2}\right\}$ where $R_{1}$ and $R_{2}$ are some positive number with $R<R_{1}<R_{2}$ and $t_{1}<t_{2}<\cdots \rightarrow \infty$. And there is a point $\left(x_{0}, k x_{0}\right)$ with $R_{1}<\left|x_{0}\right|<R_{2}$ such that in any neighborhood of $\left(x_{0}, k x_{0}\right)\left\{F_{t_{i}}\right\}_{i=1,2, \cdots}$ has no convergent subsequence. From Proposition 2.4, the case (a) does not occur. Then $\left\{F_{t_{i}}\right\}_{i=1,2, \cdots}$ diverges compactly to an algebraic curve $C$. Since $\left\{y=k x, R_{1}<|x|<R_{2}\right\} \cap\left(C \cup L_{\infty}\right)$ is a set $P$ of finite points for almost $k$ and $\left\{F_{t_{i}}\right\}_{i=1,2, \cdots}$ diverges compactly to $P$, such sequence have a convergent subsequence to a point of $P$. It is a contradiction.

Problem 4.2 The key lemmas of Theorem 4.1 are Proposition 2.3 (little Picard theorem) and Proposition 2.4 (Montel's theorem) in two-dimensional case. Since those $n$-dimensional case version are obtained in (Adachi, 2009, 2013), we think that there may be some n-dimensional case version of Theorem 4.1.

\section{References}

Adachi, Y. (1995). A generalization of the big Picard theorem. Kodai Math. J., 18, $408-424$. http://dx.doi.org/10.2996/kmj/1138043480

Adachi, Y. (1997). On the relation between tautly imbedded space modulo an analytic subset $S$ and hyperbolically imbedded space modulo S. Publ. RIMS, Kyoto Univ., 33, 385-392. http://dx.doi.org/10.2977/prims/1195145321 
Adachi, Y. (2009). A generalization of the little Picard theorem. J. Math. Anal. Appl., 354, 96-98. http://dx.doi.org/10.1016/j.jmaa.2008.12.056

Adachi, Y. (2013). On the relation between a taut manifold $X$ modulo $\Delta_{X}$ and hyperbolic modulo $\Delta_{X}$ which is contained in a hypersurface of $X . J . M . R ., 5(2), 39-41$. http://dx.doi.org/10.5539/jmr.v5n2p39

Julia, G. (1919, 1920). Sur quelques propriétés nouvelles des fonctions entières ou méromorphes. Ann. École Norm. Sup., 36, 93-125. Ibid. 37, 165-218.

Kobayashi, S. (1998). Hyperbolic complex spaces, Grundlehlen der mathmatishen Wissenschaften 318. Berlin, Heiderberg: Springer-Verlag. http://dx.doi.org/10.1007/978-3-662-03582-5_1

Tu, Z.-H. (1996). On the Julia directions of the value distribution of holomorphic curves in $\mathbf{P}^{n}$. Kodai Math. J., 19, 1-6. http://dx.doi.org/10.2996/kmj/1138043541

\section{Copyrights}

Copyright for this article is retained by the author(s), with first publication rights granted to the journal.

This is an open-access article distributed under the terms and conditions of the Creative Commons Attribution license (http://creativecommons.org/licenses/by/3.0/). 